\title{
Derivados denominales en -ble en español moderno y contemporáneo
}

\author{
Denominal derivatives in -ble in modern \\ and contemporary Spanish
}

\section{RAFAEL GARCÍA PÉREZ}

\author{
Universidad Carlos III de Madrid \\ Correo electrónico: rafael.garcia.perez@uc3m.es
}

\begin{abstract}
El siguiente artículo completa el panorama general, trazado en dos trabajos precedentes, de la evolución en español de un grupo de palabras un tanto marginal de la morfología histórica: los derivados denominales en -ble. En él se muestra cómo a partir del siglo XVIII se produce un importante cambio en los patrones de formación de este tipo de unidades respecto a la Edad Media y los Siglos de Oro. Si en estas épocas anteriores existía una gran dependencia de ciertos modelos heredados del latín clásico y, sobre todo, del latín tardío, poco a poco, a partir del siglo XVIII, nos encontramos con una sustitución de estos modelos por una tendencia más poderosa a la incorporación de préstamos, en muchos casos aleatorios, procedentes de lenguas modernas, especialmente el francés y el inglés.
\end{abstract}

Palabras clave: morfología histórica, Edad Media, Siglos de Oro, español moderno, español contemporáneo,

This paper completes the author's research on the evolution of a minor word group within Spanish historical morphology and word-formation: the denominal derivatives in -ble. We show that an important change took place from eighteenth century onwards. Whilst in the Middle Ages and the Golden Age, as it was demonstrated in two previous studies, the formation of these lexical units followed some patterns of word-formation inherited from Classical and Post-classical Latin, from the eighteenth century onwards these patterns have been replaced by a more powerful trend: the borrowing of loanwords from other modern languages, especially French and English.

Key words: Historical Morphology, Middle Ages, Golden Age, Modern Spanish, Contemporary Spanish 


\section{INTRODUCCIÓN}

En dos artículos precedentes (García Pérez, 2014a y b) estudié la formación de derivados en -ble a partir de bases sustantivas en la Edad Media y en los Siglos de Oro. Si la mayor parte de los estudios dedicados a la morfología histórica (Alvar y Pottier, 1983: 397) ponen de manifiesto que la gran mayoría de los derivados creados con este sufijo son deverbales, como sucede en nuestros días, se ha mencionado también que existió la posibilidad, mucho más restringida, de que -ble seleccionara, además, bases sustantivas (vid. Pharies, 2002: 137-138 y Val Álvaro, 1981). El problema es que en esos estudios no se había determinado con profundidad en qué condiciones se ha producido esa selección y qué causas han podido favorecerla ${ }^{1}$.

De ahí que mis anteriores trabajos buscaran sentar las bases para una explicación de la peculiar selección de bases nominales por parte del sufijo -ble y lo hiciera en esos periodos fundamentales de la historia de nuestra lengua que abarcan desde sus orígenes hasta finales del siglo XVII. Los resultados de mi investigación pusieron de manifiesto que contamos con un cierto número de formaciones que no podemos considerar simples anomalías morfológicas aleatorias y aisladas, sino restos de tendencias más profundas, generadas o acentuadas en muchos casos en el latín postclásico o tardío, que no llegaron a cuajar y, entre ellas, fundamentalmente, la creación de nuevos derivados pleonásticos en -bilis a partir de adjetivos relacionales en -alis o -ilis. Se trata de una tendencia solo apuntada en el latín clásico (como parece demostrar la existencia de exitiabilis junto a exitialis < ExITIUM, Gaffiot, 1934) que en el latín postclásico y tardío se acentuó, lo que permite rastrear una serie de préstamos interesantes del castellano medieval y áureo (amigable, racionable, vecinable, etc.) y algunas formaciones romances que podrían explicarse por imitación de las latinas (caballerible, entrañable...). También habíamos de contar, debido a la misma influencia, es decir, los modelos latinos postclásicos y tardíos, con la tendencia más restringida a una creación de derivados causativos (temorible, pavorible) o, en menor medida, activos (bonancible) a partir de sustantivos predicativos, e incluso algunos derivados a partir de bases concretas que implican una interesante reinterpretación sintáctico-semántica (fondable, pectible).

En este trabajo, siguiendo con el estudio de los derivados denominales en -ble, me centraré en esos últimos periodos en que tradicionalmente se ha dividido la historia del español: los llamados periodos moderno y contemporáneo (desde el siglo XVIII hasta la época actual). Estos periodos finales de andadura de nuestra lengua, como veremos, resultan de especial interés para nosotros, pues lejos de reflejar una simple continuidad de las tendencias morfológicas anteriores, nos muestran importantes novedades que es necesario analizar en su contexto histórico ${ }^{2}$.

\footnotetext{
1 La RAE (2009: 558-559) tampoco nos da ninguna clave. Se limita a afirmar que son escasos los adjetivos que se ajustan a la pauta $N$-able, de los que cita cuatro ejemplos, y explica el problema que plantea la determinación del sufijo en el grupo de bases sustantivas que designan cargos, profesiones o dignidades (al que me referiré en el apartado 3.6.).
}

2 Dejo fuera de este trabajo aquellos derivados que formalmente no pueden considerarse denominales 


\section{RESTOS DE LOS MODELOS PRINCIPALES MÁS ANTIGUOS}

Si en García Pérez 2014a y b, ya se mostró que, en un primer momento, la mayor parte de las formaciones en -ble con base sustantiva son préstamos del latín postclásico o tardío construidos como variantes expresivas de derivados relacionales en -alis o -ilis (amicalis > amicabilis > amigable, vicinalis > vicinabilis > vecinable, etc.), o bien creaciones romances que imitan este tipo de formaciones (caballeril > caballerible), a partir del siglo XVIII asistimos a un claro agotamiento de este patrón.

No obstante, ello no significa que no aparecieran nuevos adjetivos, aunque escasos, adaptados a esta tendencia lexicogenésica más antigua como resultado de una cierta inercia histórica. El caso más evidente es el adjetivo manuable. Lo encontramos ya en textos de principios del siglo $\mathrm{XIX}^{3}$ :

En caso de que sean muy considerables, será conveniente llevarlos formados en distintos trozos, separando las acémilas de los carros y los pesados de esta clase de los ligeros y manuables (1826, Evaristo San Miguel, Elementos del arte de la guerra).

Aunque el $D E C H$ lo considera un derivado formado en español y avanza la hipótesis de que se trataría de un cruce entre manual y manejable, lo cierto es que, esta forma existía en latín tardío. Aunque no lo recojan los glosarios dedicados al latín medieval (Blatt, 1957-1965, Diefenbach, 1857, 1867, Du Cange, 1883-1887, o Maigne d'Arnis, 1890), lo encontramos en algunos documentos de esa época, como en el siguiente párrafo de un manuscrito inglés del siglo X:

[...] arripiensque lapidem manuabilem, et in praetitulatum projecerat [...] (Stubbs, W. (ed.) (2012): Memorials of Saint Dunstan, archbishop of Canterbury, Cambridge University Press, p.455).

\footnotetext{
en sentido estricto. Es el caso de fisible, que el DRAE parece interpretar como un derivado de fisión, pero que en su origen remoto hemos de ligar al verbo latino findere. Es cierto que no aparece en los diccionarios latinos, clásicos o postclásicos y tardíos, pero tenemos el derivado fissilis sobre la raíz participal de findere (Gaffiot, 1934). Fissible aparece ya en inglés como sinónimo de fissile < FISsiLIs al menos desde el siglo XIX (a pesar de que no se recoge en el $O E D$, contamos con interesantes testimonios en algunas obras: vid., por ejemplo, McLeod, N. (1831): Dictionary of Gaelic Language, Glasgow, Hutchison \& Brookman, p. 537 s.v. so-sgoilte). Más problemático es el caso de maleable, que algunos consideran derivado de malleus 'martillo' a través del francés $(D E C H)$. Aunque A. Rey (1995) señalaba que no existía el verbo malleare, lo cierto es que este verbo lo incluye Diefenbach (1867) en su glosario de voces medievales.
}

3 Cito los datos del CORDE. Corominas y Pascual (1980-1991), en su diccionario etimológico (que citaré en adelante como DECH), nos ofrecen un testimonio de 1791. 
Se entiende así que el Diccionario universal español-latino de Valbuena, al definir el adjetivo manual, recogiera también como equivalente el derivado manuabilis junto a los adjetivos manuarius y tractabilis:

Manual. adj. Lo que cabe en la mano y se puede manejar fácilmente. Manuarius, $a$, um; manuabilis, tractabilis, $e$, is.

Es una forma, pues, que se adapta perfectamente al modelo tradicional de derivación redundante sobre adjetivos ya existentes en -alis, -ilis (García Pérez, 2014a): en este caso, nos hallamos, claro está, ante una variante pleonástica tardía de manualis, ya existente en latín clásico. El carácter culto de manuable y su incorporación tardía a la lengua han hecho que haya quedado relegado, en general, al ámbito puramente formal. Su frecuencia de uso es particularmente baja. Además, se trata de una palabra probablemente de origen peninsular, pues fue mucho más habitual en España que en América hasta los años 70; de hecho, el corpus solo nos muestra, hasta esa fecha, ejemplos aislados en Argentina, Cuba, Uruguay y Ecuador. Desde los años 80, sin embargo, asistimos a una mayor extensión por el continente americano y a una reducción de su empleo en Espańa.

Por otro lado, es también cultismo latinizante el derivado futurible (latín FUTURIBILIS). Más restringido desde un punto de vista diastrático, se introduce en los textos filosóficos peninsulares hacia mediados del siglo pasado con carácter de adjetivo muy pronto sustantivado:

Rehabilitando un viejo vocablo debido a una genial invención de Suárez, llamaremos, no futuro, sino futurible a aquello para lo cual se posee nuda potencia, pero cuyas posibilidades son aún inexistentes (1932-1944, Xavier Zubiri, Naturaleza, Historia, Dios, ed. Diego Gracia).

De nuevo nos hallamos ante un término del latín tardío (no aparece en diccionarios como Gaffiot, 1934), extendido por las obras filosóficas al menos desde el siglo XVII

[...] ergo nec rem esse praeteribilem idem est ac esse futuribilem (Lobkowitz, J.C. (1642): Rationalis et realis philosophia, Lovaina, Everard de Wirt).

Su carácter especializado no impidió que saltara a la lengua general, utilizando como trampolín, en primer lugar, la literatura:

Las dos, madre e hija, habían sido poseídas en su desnudez, en su futurible virginidad por la luz de junio... (1976, Rosa Chacel, Barrio de Maravillas).

\footnotetext{
$4 \quad$ Es posible que estuviera ya en circulación antes, aunque no he encontrado ejemplos en los textos consultados ni en los diccionarios dedicados al latín tardío y medieval. Sí aparece en Ramminger (2005).
} 
La prensa también se hizo eco de este nuevo término a partir de los años 90, lo que contribuyó a una cierta expansión por otros contextos formales:

Sin hacer mucho ruido, algunos bancos centrales llevan tiempo efectuando acopio de oro como parapeto frente a un futurible colapso de la economía mundial. (Blog, 2012, CORPES XXI)

No está de más señalar que en todo momento ha constituido un uso fundamentalmente peninsular, como pone de manifiesto el CORPES XXI. Las variedades americanas apenas lo han tenido en cuenta; de hecho, solo contamos con ejemplos puntuales en Argentina, Ecuador y México.

\section{RESTOS DE MODELOS MARGINALES ANTERIORES}

Escasas son las creaciones de derivados denominales en -ble del propio castellano. Se trata de formaciones particulares que, como en el apartado anterior, responden a otros modelos existentes en épocas anteriores, en este caso menos extendidos. Como tuve ocasión de poner de manifiesto en mis trabajos precedentes, las creaciones autóctonas basadas en estos patrones seguían también desde la Edad Media modelos latinos especialmente desarrollados en época tardía.

Uno de ellos nos presentaba al sufijo -ble dotado de una función de transpositor sintáctico-semántico capaz de integrar un sustantivo concreto en una nueva relación predicativa (recordemos los ejemplos de fondable y pectible, García Pérez, 2014 a y b). Así se creará también, en el siglo XIX, el sustantivo maderable, aplicado a los árboles o plantas, con el sentido de 'que produce madera'. Aunque existía el verbo maderar desde mucho antes, este era una variante de enmaderar, entendido como 'cubrir con madera' o incluso 'construir con madera', de modo que parece claro que maderable solo puede analizarse como un denominal.

El suelo es pobre á pesar de sus minas, (hierro, cobre y plata), y bosques maderables, siendo las principales producciones (1865, Manuel Merelo, Nociones de geografía descriptiva, ed. Librería de San Martín).

Desde el primer momento, este adjetivo pareció instalarse con éxito en el uso, pues los ejemplos son numerosos desde finales del siglo XIX hasta nuestros días. No obstante, maderable sufrirá una evolución interesante que lo pone en contacto, indirectamente, con los adjetivos creados según el antiguo modelo pleonástico respecto a derivados en -al (más raramente en -ero, como señalé en García Pérez, 2014a). A partir de principios del siglo $\mathrm{XX}$ adquiere, pues, un sentido relacional aislado que se extenderá con más fuerza desde finales de la misma centuria, aun cuando no llegue a arraigar completamente. Entra así en competencia con maderero: 
[...] el terreno tiene también a veces decisiva importancia en la producción de la hierba, pues conocemos castañares bravíos explotados por su aprovechamiento maderable (1921-1944, Ezequiel González Vázquez, Alimentación de la ganadería y los pastizales españoles, Ediciones técnicas).

La jurisdicción de dicho Comité corresponde, por cierto, a la zona donde se concentra alrededor del 50\% de la actividad maderable (1996, CREA).

Esta confluencia con maderero, quizás, ha llevado a que este último también haya podido utilizarse, puntualmente, con el significado inicial de maderable.

[...] los bosques de árboles madereros plantados por el rey D. Dionisio de Portugal significaban una preparación (1946-1952, Manuel Ballesteros Gaibrois, Historia de América, ed. Ediciones Pegaso).

Otro modelo permitía la creación de derivados a partir de bases predicativas. A este grupo pertenecería el adjetivo presidiable , pues, aunque el $D R A E$ parece hacer referencia a la acepción locativa de la base como punto de partida para su creación (centro penitenciario), bien podría ser que en realidad se hubiera partido de su sentido más abstracto de 'pena de privación de libertad', lo que justificaría una paráfrasis del tipo 'que merece ser condenado a (una pena de) presidio' y no tanto 'que merece estar en presidio' (DRAE s.v. presidiable). Desde ese punto de vista, la base estaría constituida por un sustantivo predicativo y el sufijo -ble mantendría, sin demasiada violencia, su sentido pasivo tradicional. Se explicaría así mejor que los primeros ejemplos, situados cronológicamente a principios del siglo XX, nos muestren como primer argumento o argumento sujeto tanto sustantivos designadores de autores de delitos como los delitos mismos:

En Whitechapel seguían abundando las gentes presidiables: ladrones, asesinos y comerciantes (1929-1933, Enrique Jardiel Poncela, Amor se escribe sin hache. Novela casi cosmopolita, ed. Roberto Pérez).

[...] y lo de menos es que el ganador sea el propio hombre abocado a la paternidad o los antepasados de éste; ni que sea fruto de una vida de probidad o de una rapiña presidiable (1919-1929, Gregorio Marañón, Ensayos sobre la vida sexual, ed. Espasa-Calpe).

El adjetivo se ha mantenido con poca fuerza en el uso lingüístico; de hecho, ni en el CREA ni en el CORPES XXI se atestiguan ocurrencias. No obstante, sí es posible

\footnotetext{
5 Por supuesto, hemos de rechazar, por razones semánticas, la conexión entre presidiable y presidiar, pues este verbo tenía el significado de 'guarnecer con soldados alguna plaza o castillo' (ref. Autoridades, cito por el NTLLE (Nuevo tesoro lexicográfico de la lengua española).
} 
encontrar ejemplos recientes en Internet, más en libros que en la prensa escrita. Se puede decir que este vocablo ha llegado a nuestros días, más bien, como un arcaísmo restringido a los contextos formales.

No parece haber sobrevivido el patrón que permitía la formación de derivados causativos a partir de bases predicativas (temorible, pavorible). El adjetivo deshonrible, que el corpus nos ofrece desde el siglo XIX, formado sobre la base deshonra, es solo un espejismo, al que contribuye el $D R A E$ al recogerlo por primera vez en 1884 . Lo define como 'despreciable' ${ }^{6}$, lo que supone una pequeña evolución semántica, perfectamente regular, por otra parte, respecto al significado previsible según su etimología y su proceso morfológico de construcción, que respondería a este patrón de sustantivo predicativo + ble $=$ resultado causativo:

[...] volvámoslo á doblar por un procedimiento inverso de desevolución, y así se verá el origen deshonrible de esta especie simia llamada hombre, que Dios sacó de la nada hace tres ó cuatro días, según Moisés, y hace millones de años, según los naturalistas del Ateneo (1891, Ramón de Campoamor, La metafísica y la poesía ante la ciencia moderna, ed. Felipe González Rojas).

Parece que en este caso el corpus -al igual que el DRAE- revela, sencillamente, una importante laguna; en realidad, este adjetivo debía de ser bastante más antiguo. Se remonta, al menos al siglo XVII, como queda patente en el siguiente ejemplo:

Pues si duerme (dizen) no tendrá peligro su achaque: como si vn dormir ocioso en las obligaciones, no sea una muerte deshonrible de la opinion. (Estrada Gijón, J. (1661): Sermones varios, ed. Juan de Calatayud y Montenegro, p.435)

La antigüedad de esta formación permite integrarlo con mayor rigor en los patrones lexicogenésicos tradicionales. Es muy probable que su extensión por la lengua, especialmente la lengua culta, no fuera muy amplia, lo que explicaría por qué aparece reflejado en una etapa tan tardía en el CORDE.

Es curioso constatar, sin embargo, que la creación de deshonrible no dio lugar a la formación de su opuesto morfológico honrible o, en todo caso, no ha dejado restos en los textos ${ }^{7}$.

\footnotetext{
$\overline{6}$ Junto a este adjetivo aparecía también en la definición 'sin vergüenza', que no he logrado localizar en el corpus.

Naturalmente, en los textos que yo he consultado, lo cual no supone que no puedan aparecer testimonios procedentes de otras fuentes. En todo caso, parece posible afirmar que, si existió, su uso debió de ser bastante menos frecuente que el de deshonrible.
} 


\section{INNOVACIONES}

La mayor parte de las formaciones denominales en -ble son, sin embargo, simples préstamos procedentes de otras lenguas, especialmente, el francés y el inglés. Se trata de un fenómeno que no había tenido repercusión en siglos anteriores, caracterizados por una dependencia casi exclusiva, como tuve ocasión de señalar en García Pérez, 2014b, de los modelos latinos postclásicos y tardíos; de ahí que podamos hablar de una ruptura considerable en los mecanismos de introducción de este tipo de derivados en nuestra lengua. La razón de esta preponderancia de las lenguas modernas frente al latín ha de buscarse en el nuevo prestigio que el francés y el inglés llegaron a adquirir a partir del siglo XVIII.

El adjetivo viable se introduce en español en la segunda mitad del siglo XIX para designar la capacidad de seguir viviendo, especialmente aplicado a los nińos recién nacidos. El origen se encuentra en el francés viable, derivado del sustantivo vie 'vida', cuyos primeros testimonios se remontan al siglo XVI (A. Rey, 1995). A partir de él se formó, aunque bastante más tarde, el derivado viabilité (principios del siglo XIX), que pasará al español con la forma viabilidad. Dadas las fechas de incorporación a nuestra lengua, se entiende que aparezcan casi simultáneamente tanto viable como viabilidad:

Se comprende que todas estas anomalías influirán en la viabilidad del feto, aunque no con igual eficacia y que obedecen á suspensiones en la evolución de los arcos aórticos (1870-1901, Julián Calleja y Sánchez, Compendio de anatomía descriptiva $y$ de embriología humanas, II).

[...] incapaces para producir hijos viables y para gustar las delicias del amor, aquellos dos niños pronuncian la sentencia definitiva sobre toda su vida (1873, Serafín Álvarez, El Credo de una religión nueva, ed. José Esteban).

La existencia de estos dos derivados hizo posible la formación temprana de sus antónimos. De ahí que junto a viabilidad encontremos también inviabilidad:

Cuando además de esta anomalía existe rotura de las paredes abdominales y el corazón se escapa por ella, determina la inviabilidad del feto (1870-1901, Julián Calleja y Sánchez, Compendio de anatomía descriptiva y de embriología humanas, II).

La aparición más tardía de inviable (segunda mitad del siglo XX), ya dotado de su extensión semántica, parafraseable como 'que carece de las condiciones necesarias para existir o mantener su existencia', ha de interpretarse como una simple laguna del corpus; es posible que a ello haya contribuido el carácter excesivamente formal y técnico del vocablo. Conviene recordar que en francés ya se había difundido, sin embargo, desde 1877 (A. Rey, 1995): 
[...] y por lo mismo algo absolutamente romano, políticamente inviable fuera del mareo de vida de la comunidad romana (1950-1968, Jesús Fueyo Álvarez, Estudios de teoría politica).

Pero contamos en el propio francés con un homónimo, viable, resultado de la evolución del latín viabilis (< VIA) aplicado a los caminos ('que puede ser transitado'). Los primeros testimonios se remontan a 1790 (A. Rey, 1995). Este adjetivo constituirá la base para un nuevo derivado, viabilité, que designaba el estado de una vía (transitable), pero también el conjunto de obras necesarias en el ámbito de la construcción. El español viabilidad, que supone el previo viable, aun cuando este se haya creado en francés, es el primer ejemplo que encontramos en el corpus (finales del siglo XIX):

[...] costear los servicios públicos que corren á cargo de la hacienda municipal: instrucción, beneficencia, policía rústica y urbana, viabilidad, higiene, corrección, ornato, etc. (1898, Joaquín Costa, Colectivismo agrario en España).

A pesar de que este segundo viable aparece en el DRAE sin marca alguna, su uso es mucho menos frecuente $y$, sin duda, se halla bastante más restringido diastráticamente. Por su parte, los antónimos inviable e inviabilidad apenas se han empleado ni se emplean en nuestros días con el significado de 'intransitable' o 'imposibilidad de ser transitado'.

También es un galicismo el adjetivo carrozable. En francés aparece por primera vez en 1825 (A. Rey, 1995), como derivado del sustantivo carrosse para designar la vía o el lugar por el que pueden circular los vehículos. Su introducción en español es más bien tardía, pues hemos de esperar a la segunda mitad del siglo XX para encontrar los primeros testimonios:

Una trocha carrozable de treinta y cuatro kilómetros, estrecha, tortuosa, polvorienta [...] (1971, Leónidas Castro Bastos, Paisajes natural y cultural del Perú).

Sorprende el escaso número de ejemplos ofrecidos por el CORDE. Podríamos pensar que se trata, pues, de una palabra poco frecuente, de carácter formal, y reservada principalmente a las variedades americanas del español, principalmente Perú y Ecuador (como pone de manifiesto el CORPES XXI, que confirma la marcación geográfica del DRAE). Si en Internet los ejemplos son más numerosos, un estudio de su tratamiento en la prensa escrita parece confirmar que su mayor expansión, efectivamente, se sitúa en América, aunque puntualmente podemos encontrar también este vocablo en ciertos periódicos españoles (El Mundo, La Vanguardia), especialmente cuando retoman noticias procedentes de la prensa americana.

Un uso peculiar de carrozable -y bastante más moderno- traduce la expresión francesa à carrosser aplicada a los vehículos (carrosser significaría aquí 'dotar a un vehículo de carrocería', tal como lo define el TLF). Se trata de un salto semántico muy interesante 
que pone en conexión morfosemántica - de un modo más bien artificial- el antiguo adjetivo carrozable con el sustantivo carrocería. Aunque no aparecen ejemplos en los corpus, la prensa escrita se ha hecho eco, en algunos casos, de este nuevo uso, no recogido, por cierto, en el DRAE:

La distancia desde el paragolpes delantero hasta la parte más avanzada de la caja de carga es menor en la nueva gama, con lo que se dispone de una mayor longitud carrozable (El Mundo, Motor \& viajes, 6-5-2000).

Mayor presencia en los corpus ha tenido el adjetivo penible, formado previamente en francés $(<$ peine), concretamente en el siglo XII, y aplicado, en un primer momento, a las cosas que se hacen con esfuerzo; posteriormente pasó a significar 'difícil de aguantar' (A. Rey, 1995), significado que encontramos en los primeros ejemplos del español (siglo XVIII), probablemente como consecuencia de las traducciones de obras francesas llevadas a cabo en esa época con cierta profusión:

La caza de la gamuza es muy penible y difícil [...] (Enciclopedia metódica. Historia natural de los animales traducida del francés al castellano por D. Gregorio Manuel Sanz y Chanas, 1788, p. 116).

Este galicismo está bastante menos limitado, desde el punto de vista cronológico, de lo que parecería a primera vista. De hecho, se puede decir que ha tenido un cierto arraigo en la lengua, especialmente entre los hablantes cultos, pues lo encontramos, aunque no sea muy frecuente, hasta bien entrado el siglo XX (el CREA, por ejemplo, nos ofrece un testimonio de la lengua oral).

A esta penible agitación de Juanita se contraponía en su alma otra agitación dulcísima, otro sentir [...] (1895, Juan Valera, Juanita la Larga, ed. Enrique Rubio).

Sin embargo, ha quedado prácticamente excluido de la prensa escrita en nuestros días, lo cual no es de extrañar dada la política de limpieza de galicismos de la mayor parte de los gramáticos puristas desde el siglo XVIII.

Por su parte, el adjetivo equitable parece haber sido una mera moda dieciochesca $y$, por tanto, bastante efímera, producto de la profunda influencia francesa en el léxico español característica de ese momento histórico. Si en francés se creó ya en el siglo XVI (A. Rey, 1995), los ejemplos españoles de este préstamo son escasos y se limitan al siglo de las luces:

Que se establecerá nuevo comercio para que se conceda la libertad necesaria conservando la más equitable armonía (1763, Artículos [Documentos indispensables para la verdadera historia de Filipinas], ed. Eduardo Navarro). 
De entre los préstamos procedentes de las lenguas modernas, llama especialmente la atención el adjetivo inhospitable. Se trata de un préstamo del inglés, donde encontramos también el opuesto morfológico hospitable; ambos vocablos se tomaron, a su vez, del antiguo francés. Es muy probable que el origen remoto se halle en una variante pleonástica tardía del latín clásico hospitalis 'hospitalario's. Los primeros testimonios en inglés se remontan al siglo XVI y su empleo continúa hasta finales del siglo XIX (OED, s.v. inhospitable $)^{9}$. Es precisamente en el siglo XIX, una vez desaparecido su equivalente en la lengua francesa, cuando lo encontramos en los textos españoles:

La emperatriz levantó un hermoso palacio en aquel desierto inhospitable y al punto la imitaron todos sus favoritos y grandes seńores de la corte (1847-1857, Juan Valera, Correspondencia, ed. Imprenta alemana).

Es curioso, sin embargo, que no contemos con ningún ejemplo de su opuesto morfológico hospitable, bien atestiguado, como he apuntado más arriba, en inglés. Ello parece poner de manifiesto que nos hallamos ante un préstamo que no llegó a arraigar en el léxico espańol. De hecho, los testimonios conservados son bastante escasos y no superan la barrera cronológica del siglo XIX. Es significativo, a ese respecto, que no se encuentren ejemplos en la prensa actual.

También por influencia de otros idiomas se ha creado en español un nuevo paradigma derivativo en el que el sufijo -ble se adjunta a bases nominales predicativas pertenecientes a la clase sintáctico-semántica cuyos elementos designan cargos públicos (estos adjetivos ya aparecen citados en Rainer, 1999, y los retoma la RAE, 2009: 558). En ese sentido, el derivado adjetivo, manteniendo el significado pasivo del sufijo, hace referencia al candidato a dicho cargo. Se trata de un fenómeno lingüístico relevante para la historia de la lengua, porque no nos hallamos ante simples creaciones morfológicas aisladas, sino ante la construcción de una nueva regla derivativa que va a alcanzar especial aceptación en el español actual. La historia de estos adjetivos no deja de ser interesante. Su origen inmediato hemos de situarlo, por razones cronológicas, en el adjetivo francés papable, italianismo, a su vez, del siglo XVI (TLF, A. Rey, 1995): papabile. El paso al español es relativamente tardío, pues hemos de esperar hasta el siglo XVIII:

[...] lo uno, porque tienen miedo á los Borbones, y lo otro, porque los papables no quieren cortarse la esperanza tan de rondon (1769, José Nicolás de Azara, Cartas de Azara al ministro Roda).

\footnotetext{
$8 \quad$ El OED, s.v. hospitable, plantea la posibilidad de que se trate de un deverbal formado sobre la base tardía hospitare. Aunque tal base debió de existir (al menos la recoge Diefenbach, 1867), bien podría tratarse de un caso más de derivación pleonástica a partir de un relacional ya existente, tanto más cuanto que hospitalis había tenido los mismos usos contextuales.

9 En francés podemos considerarlo desaparecido desde el siglo XVII (al menos, la base de datos FRANTEXT no nos proporciona ningún ejemplo desde esa fecha).
} 
De ahí que en el siglo XIX apareciera también el adjetivo ministrable, si bien es difícil determinar si se trata de un nuevo galicismo o, por el contrario, es ya una creación autóctona, ya que en francés su formación es casi simultánea (1885).

Si esta merma procedía de los banquetes y otras parecidas travesuras con que el marqués trataba de hacerse visible, y hasta ministrable, entre los hombres políticos de mayor talla [...] (1888, José María Pereda, La Montálvez, ed. Imprenta de M. Tello).

[...] mientras que dividiéndose en hatillos ó cofradías de corto personal, irían todos entrando en el comedero, y hasta los gatos serían ministrables (1910, Benito Pérez Galdós, Amadeo I, ed. Páez Perlado y Cía).

Se puede afirmar, pues, que en estos momentos el paradigma estaba solo anunciado. Será a partir de la segunda mitad del siglo XX cuando se constituya propiamente. Siguiendo el modelo de estos dos adjetivos encontraremos ya, por orden de antigüedad y frecuencia, los derivados presidenciable (1977) alcaldable (1978), rectorable (1996) y otros que no han dejado restos en el corpus, pero que es posible rastrear por otros medios, como decanable y cardenalable, fáciles de encontrar en Internet. La posibilidad de crear nuevos adjetivos siguiendo este patrón aún está abierta.

\section{Conclusión}

Con este artículo se ha completado el panorama general de la evolución en español de los derivados denominales en -ble. En él se ha puesto de manifiesto que los periodos moderno y contemporáneo suponen un cambio importante en los patrones de formación de este tipo de unidades léxicas. Si en la Edad Media y los Siglos de Oro existía una gran dependencia de ciertos modelos heredados del latín clásico y, sobre todo, del latín tardío, poco a poco, a partir del siglo XVIII, nos encontramos con una sustitución de estos modelos por una tendencia más poderosa a la incorporación de préstamos, en muchos casos aleatorios, procedentes de lenguas modernas, especialmente el francés y el inglés. De ahí la dificultad de hablar de modelos o patrones lexicogenésicos en sentido estricto en estas centurias finales de la historia de nuestra lengua; ello ha redundado, sin duda, en la imagen de arbitrariedad que parece ofrecernos hoy en día este paradigma morfológico.

\section{OBRa CITADAS}

Alvar, M. y Pottier, B. 1983. Morfología histórica del español. Madrid: Gredos. Blatt, F. 1957-1965. Novum Glossarium Mediae Latinitatis ab anno DCCC usque ad an- 
num MCC. Edendum curavit Consilium Academiarum Consociatarum. Copenhagen: Munksgaard.

Corominas, J.y Pascual, J. A. 1980-1991. Diccionario critico etimológico castellano e hispánico. Madrid: Gredos.

Diefenbach, L. 1857. Glossarium latino germanicum mediae et infamae aetatis. E codicibus manuscriptis et libris impressis concinnavit. L. Diefenbach: Frankfurt.

Diefenbach, L. 1867. Novum glossarium latino germanicum mediae et infimae aetatis. J. D. Sauerländer's Verlag: Frankfurt.

Du Cange et al. 1883-1887. Glossarium media et infima latinitatis. Niort: L. Favre.

Gaffiot, F. 1934. Dictionnaire latin-français. Paris: Hachette.

García Pérez, R. 2014a. "Un acercamiento a los derivados denominales en -ble en castellano medieval". "Llaneza". Estudios dedicados al profesor Juan Gutiérrez Cuadrado. M. Bargalló Escrivá, M. Pilar Garcés Gómez y C. Garriga Escribano (eds.). Anexos de la Revista de Lexicografía 23. 69-80.

García Pérez, R. 2014b. "Un acercamiento a los derivados denominales en -ble en los Siglos de Oro". Revista Linguae 1. 137-151.

Maigne d'Arnis, W. H. 1890. Lexicon manuale ad scriptores mediae et infimae latinitatis, ex glossariis Caroli Dufresne, D. Ducangii, D.P. Carpentarii, Adelungii, et aliorum, in compendium accuratissime redactum. Paris, apud Garnier Fratres.

Pharies, D. 2002. Diccionario etimológico de los sufijos españoles. Madrid: Gredos.

Rainer, F. 1999. "La derivación adjetival". Gramática descriptiva de la lengua española. Bosque, I. y Demonte, V. (dirs.). Madrid: Espasa Calpe. 4595-4643.

Ramminger, J. 2005-. Neulateinische Wortliste. Ein Wörterbuch des Lateinischen von Petrarca bis 1700 [en línea]. Disponible en: <www.neulatein.de> [Consulta: junio de 2013).

Real Academia Española: Banco de datos (CORDE: Corpus diacrónico del español) [en línea]. Disponible en: <http://www.rae.es> [Consulta: junio de 2014].

Real Academia Española: Nuevo tesoro lexicográfico de la lengua española [en línea]. Disponible en: <http://www.rae.es> [Consulta: junio de 2014].

Real Academia Española (2009): Nueva gramática de la lengua española, Madrid: Espasa.

Val Álvaro, J. F. 1981. "Los derivados sufijales en -ble en español". Revista de Filología Española 61. 185-198. 
